\title{
DIVERSITY AND STATUS OF BRYOPHYTES IN PANCH POKHARI REGION OF THE NORTHERN SINDHUPALCHOK DISTRICT OF CENTRAL NEPAL
}

\section{N. Pradhan}

\section{ABSTRACT}

This is the first exploration of bryophytes in Panch Pokhari of Sindhupalchok district of central Nepal vertically ranged from 850 to $4300 \mathrm{~m}$ asl. Of the total record of 151 species, 60 species were categorized as rare in status and two species Jamesoniella automnalis (D.C.) Steph. and Campylopus rechardii Brid were recorded as threatened species.

Key Words: altitude, rare, bryoflora, species richness

\section{INTRODUCTION}

The changing pattern of climate along the altitudinal gradient is the characteristic feature of Nepal. This feature has created interesting and diverse ecosystems at various vertical pockets where number of habitat specific flora and fauna are accommodated under the influence of macro and micro-climatic conditions.

The coverage of bryophytes varies within catchment types; high altitude springs support dense mats, unlike streams fed by ice and glaciers. Taxa confined to low altitudes include characteristic of humid subtropical conditions (Ormerod et al. 1994). Nepal has represented good diversity of bryoflora, which counts 1205 species hitherto. This includes 428 species of liverworts and 11 species of hornworts (Pradhan and Joshi 2009). Pradhan recorded 766 species of mosses from Nepal (Pradhan 2000). Earlier, Kattel and Adhikari (1992) compiled a list of 559 species of mosses in Nepal. Integration of complex geographical regions and varied climatic types are the vital factors to bring changes in bryofloral species at varying altitudinal pockets of this country. Grau et al. (2007) reported a unimodel relationship between species richness and altitudes for both liverworts and mosses in Nepal with maximum species richness between 2500 and 2800 $\mathrm{m}$ of elevations. Many of the endemic species were recorded from 2500 to $3000 \mathrm{~m}$ of elevation.

This study was concerned mainly to make an extensive study of bryophytes of the remote Panch Pokhari region which was an unexplored area before. This study also recorded the diversity change and species decline of this plant with addition of altitudes at $1350 \mathrm{~m}$ to $4300 \mathrm{~m}$.

\section{STUDY AREA}

Panch Pokhari, a part of the Langtang National Park stands at $27^{\circ} 36^{\prime}$ to $28^{\circ} 13^{\prime} \mathrm{N}$ and $85^{\circ}$ 2 ' to $86^{\circ} 06^{\prime}$ E. (fig. 1). Being enriched with highly valued biodiversity, interesting terrains and mind blowing peaks, this area is still beyond the reach to prosperous tourism besides having incomplete documentation of the valuable natural history existing there. 
perate climatic types from the villages of Melamchi $(850 \mathrm{~m}$ ) to Dhap ( $1300 \mathrm{~m}$ ), Botang (1600 $\mathrm{m}$ ) and Nosim (3500 m). The temperate and subtropical zones displayed rich floral components including interesting bryophyte species. The sub tropical vegetation like the Oak forest (Quercus lanata) was observed at 1000 to $2000 \mathrm{~m}$. A lower temperate Oak- Rhododendron forest constituted by Quercus lanata and Rhododendron arboreum can be seen below the village Dukhan $(2200 \mathrm{~m})$ at the dry rocky slope of Hingar Khola. The moist area in this place was dominated by Alnus nepalensis with Lyonia ovalifolia, Rubus ellipticus, Oxyspora paniculata, etc. Subalpine vegetation above the villages of Deurali to Nosim (3500-3800 m) represented Rhododendron (R. campanulatum) with associated species like Abies spectabilis, Betula utilis, Juniperus recurva, etc. The alpine zone at Panch Pokhari $(4200 \mathrm{~m})$ displayed lustrous green meadows with Rhododendron lepidotum, $R$. anthopogon, $R$. setosum, Juniperus recurva and J. indica (fig. 1).
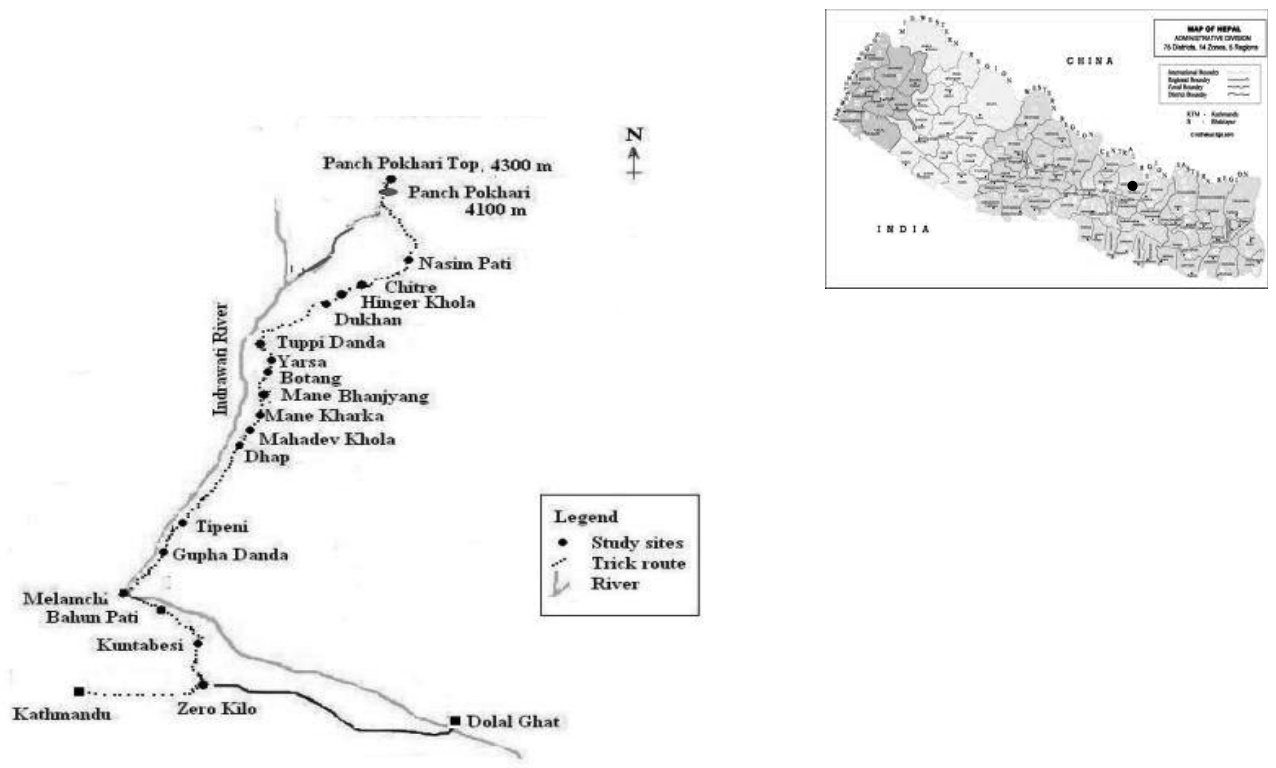

Figure 1. Survey route in the study area.

\section{MATERIALS AND METHODS}

The bryophyte specimens were collected during 2010-2011 using pocket knife and placed safely into the paper packets. The hand lens with magnifying power (5X-20X) was used for field identification. Various relevant literatures like Allen et al. (2002), Chopra (1975), Eddy (1988, 1990, 1996), Gangulee (1969-1980), So (1995), Smith (1996) and Zhu and So (1996) were consulted for identification work. The author's names were checked consulting the book by Brummitt and Powell (1992).

Quadrats of $5 \times 5 \mathrm{~m}$ were set at different elevations to measure the species richness occurring 
within each quadrat. Three quadrats were set in a gap of $500 \mathrm{~m}$ of elevation except at 800 $1500 \mathrm{~m}$ where 4 quadrats and at $4300 \mathrm{~m}$ two quadrats in a gap of $300 \mathrm{~m}$ were used. Overall 21 quadrates were set during entire study period.

Pearson's correlation coefficient ( $r$ ) was used to measure the strength of association between two variables (altitude and species of bryophytes) (Wessa 2012).

\section{RESULTS AND DISCUSSION}

Bryophyte species and their status on local level were categorized at different elevations. This study was initiated at the lowermost elevation of $800 \mathrm{~m}$ in Melamchi village of Sindhupalchok district and species record was made at every $500 \mathrm{~m}$ of elevations upto the range of $4300 \mathrm{~m}$. A detail list is provided in the appendix 1.

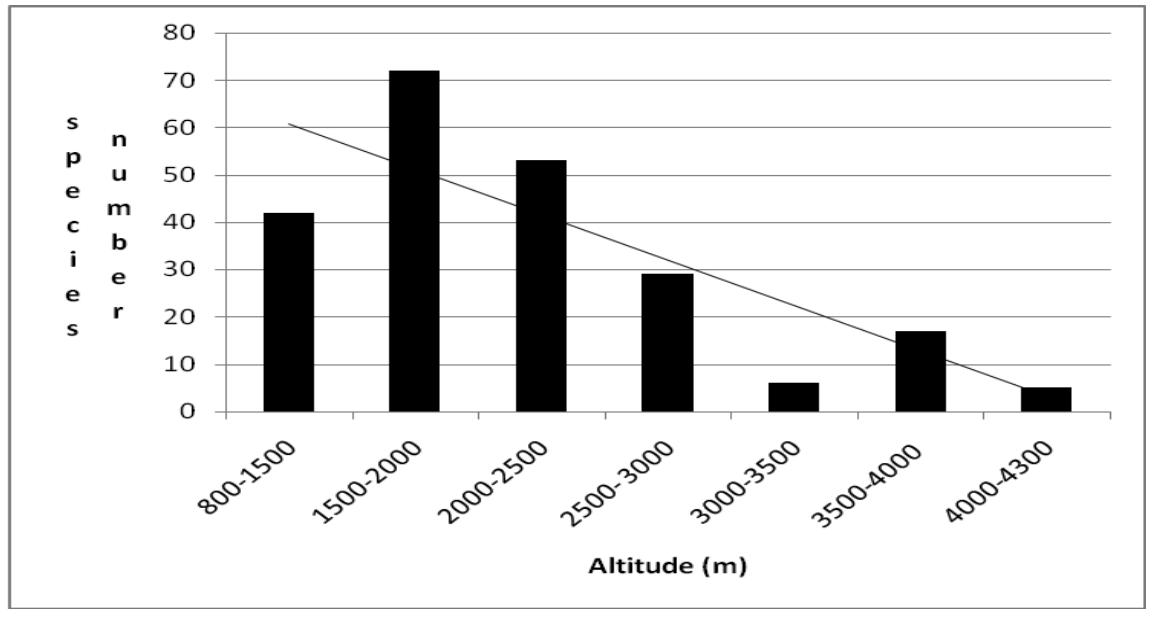

Figure 2. Number of bryophyte species at different elevations.

This study brought a list of 151 species of bryophytes under 43 families and three classes. The class Anthocerotae included 5 species, Hepaticae 48 species, and Musci 98 species. Among Hepaticae, the order Jungermanniales showed dominancy with 29 species belonging to 10 families; Metzgeriales with 3 species belonging to 3 families and thalloid liverworts (Marchantiales) with 16 species belonging to 6 families. Negative correlation $(r=-0.763)$ was found between the altitudes and species diversity of bryophytes at the vertical range of Panch Pokhari region.

Among the Musci, the dominant family was Bryaceae with the record of 17 species. Second dominant families observed were Dicranaceae and Pottiaceae with 8 and 7 species respectively. An interesting hairy capped moss called Tayloria indica (Four toothed Moss) of the family Splachnaceae was recorded in animal dung at $2460 \mathrm{~m}$ of elevation. Bryum coronatum which is a common moss at lowland Tarai was also recorded at 1200-1760 m of this region. Conservation status of every species was assessed on local level. This was done by direct count of the observed species patches at different altitudinal levels besides consulting available literatures. This brought a list of 72 rare species. Two species, like Jamesoniella automnalis (DC.) Steph. and Campylopus rechardii Brid. were assessed as very rare species (VR). Rest 
84 species ranged from common to fairly common in their status. Bryum argenteum, Funaria hygrometrica, Pohlia elongata and Entodontopsis tavoyensis were widely spread species across lowland to the Himalayan regions.

Sun et al. (2013) studies the terrestrial bryophyte diversity and community structure along an altitudinal gradient from 2001 to $4221 \mathrm{~m}$. in Gongga Mountain in Sichuan, China. The terrestrial bryophyte biomass and thickness displayed a clear humped relationship with the elevation, with the maximum around $3,758 \mathrm{~m}$. According to them, bryophyte distribution is primarily associated with the depth of litter, the air temperature and the precipitation.

According to Liu et al. (2008), the analysis of environmental factors and bark characteristics, for epiphytic bryophytes, atmospheric humidity gradient might be one of the major factors that affects the vertical distribution, and bark $\mathrm{pH}$ might be an explanation for the difference in the epiphytic cover among tree species, but bark water content has no relation to the distribution pattern. Finally, community structure of epiphytic bryophytes on the same tree species is also affected by forest types.

Bio-physical gradients like temperature, rainfall, humidity, altitude and forest canopy played controlling effect to distribute and bring species richness of bryophytes at different altitudinal pockets of Panch Pokhari region. Change in species community was also noticed at different altitudes under the influence of these gradients. Species diversity was noticed very low at 4300 $\mathrm{m}$ where favorable conditions of the bio-physical factors like temperature, humidity, rainfall, and soil were not prevailing. This study recorded a maximum diversity of 72 species at $1500-2000$ $\mathrm{m}$ of elevation where warm and humid condition, optimum amount of precipitation, favorable temperature and good forest canopy prevailed. Next factor which acted to decline species level at higher elevation of Panch Pokhari is the lack of canopy for its dependent species. Epiphytic bryoflora was completely lacking at this range due to the absence of host tree species.

An extensive study is still necessary at the southern and central parts of the Sindhupalchok district in order to bring perfectness in findings on how this plant is distributed at different altitudinal pockets on the vertical ranges under the influence of varying degrees of bio-physical gradients.

\section{ACKNOWLEDGEMENTS}

I am highly indebted to the Rufford Small Grant Foundation, UK, for sponsoring this work under its small grant program. I am thankful to Professors Bhaiya Khanal (Zoology), Natural History Museum and Dr. Mohan Siwakoti (Botany), Central Department of Botany, Tribhuvan University, for their significant involvement as members of this research work. Similarly, Dr. Sandesh Bhattarai, Nepal Academy of Science and Technology (NAST) who was also a research member in this work is highly acknowledged. Likewise, my thanks are due to supporting field researchers like, Mr. Vinod Thapa (Zoology), Mr. Amin Pun (Botany), Mr. Rajesh Tamang (Botany). Ms. Suprava Shrestha (Botany), Ms. Sanam Prajapati (Botany) and Ms. Geeta Thapa (Zoology). I am grateful to Mr. Hira Bahadur Lama of Botang (Sindhupalchok District) for his good management, help and cooperation in the field. Mr. Deb Bahadur Lama and his family are equally acknowledged for providing us accommodation and all the essential help required in the field. 
Pradhan : Diversity and Status of Bryophytes in

\section{REFERENCES}

Allen, B., D. Hall, J. Munoz, R.A. Pursell and W.R. Buck, 2002. Mosses of Central America. 90(2):1-700. Missouri Botanical Garden, St. Louis, USA.

Brummitt, R.K. and C.E. Powell, 1992. Author's of Plant Names. Royal Botanic Garden, Kew, UK. Chopra, R.S., 1975. Taxonomy of Indian Mosses. Bot. Monogr. 10. Pubs., \& Inf. Directorate, New Delhi, India.

Eddy, A., 1988. A Handbook of Malesian Mosses I. Nat Hist Mus (BM), London.

Eddy, A., 1990. A Handbook of Malesian Mosses II. Nat Hist Mus (BM), London.

Eddy, A., 1996. A Handbook of Malesian Mosses III. Nat Hist Mus (BM), London.

Gangulee, 1969-1980. Mosses of Eastern India and Adjacent regions Fasc. 1-8:1-2145, Calcutta, India.

Grau, O., J.A. Grytnes and H.J.B. Birksm, 2007. A comparison of altitudinal species richness patterns of bryophytes with other plant groups in Nepal, Central Himalaya. Journal of Biogeography, 34:1907-1915.

Kattel, L.P and M.K. Adhikari, 1992, Mosses of Nepal. Nat. Hist. Soc.Kathmandu, Nepal, pp $1-107$.

Liu, W.Q., D.X. Hua, W.Y. Fan and L.C. Yi, 2008. Analysis of environmental factors affecting the distribution of epiphytic bryophyte at Heishiding Nature Reserve, Guangdong Province [J]. Acta Ecologica Sinica, 28(3):1080-1088.

Ormerod, S.J., S.D. Rundle, S.M. Wilkinson, G.P. Daly, K.M. Dale and J. Juttner 1994. Altitudinal trends in the diatoms, bryophytes, macro-invertebrates and fish of a Nepalese river system. Freshwater Biology, 32(2):309-322.

Pradhan, N. 2000. Materials for a Checklist of Bryophytes of Nepal. British Museum, London.

Pradhan, N. and S.D. Joshi, 2009. Liverworts and Hornworts of Nepal: a synopsis. Botanica Orientalis. Journ. of PI. Sci., 6(6):69-75.

Smith, J.A .E. 1996. The Liverworts of Britain and Ireland. Cambridge University Press, Cambridge, UK., $362 \mathrm{p}$.

So, M.L. 1995. Mosses and Liverworts of Hong Kong. Vol.1. Hevenly People Depot.

Sun S-Q, Wu Y-H, Wang G-X, Zhou J. and Yu D et al., 2013. Bryophyte Species Richness and Composition along an Altitudinal Gradient in Gongga Mountain, China. PLOS ONE, 8(3):77-88.

Wessa P., 2012. Pearson Correlation (v1.0.6) in Free Statistics Software (1).23-r7), Office for Research Development and Education, URL http://www.wessa.net/rwasp_correlation.wasp/ Zhu, R.L. and M.L. So, 1996. Mosses and Liverworts of Hong Kong. Vol.2. Heavenly People Depot. 


\section{Appendix I}

Table 1. Distribution of Bryophytes at different elevations of northern Sindhupalchok district.

\begin{tabular}{|l|l|l|l|l|}
\hline SN & Families & Scientific Names & Elevation in meter & Status \\
\hline
\end{tabular}

\section{Anthocerotae}

\begin{tabular}{|l|l|l|l|l|}
\hline 1. & Anthocerotaceae & $\begin{array}{l}\text { Anthoceros chambensis } \\
\text { Kashyap }\end{array}$ & 1300 & FC \\
\hline 2. & Anthocerotaceae & Anthoceros punctatus L. & $1480-3500$ & C \\
\hline 3. & Anthocerotaceae & Anthoceros longii St. & 1450 & FC \\
\hline 4. & Anthocerotaceae & $\begin{array}{l}\text { Folioceros assamicus D. C. } \\
\text { Bhardwaj }\end{array}$ & 1300 & R \\
\hline 5. & Anthocerotaceae & Phaeoceros laevis (L.) Prosk. & 1700 & FC \\
\hline
\end{tabular}

\section{Hepaticae}

\begin{tabular}{|c|c|c|c|c|}
\hline 1. & Aneuraceae & Riccardia multifida (Linn.) Gray & $1200-2850$ & $\mathrm{FC}$ \\
\hline 2. & Aytoniaceae & $\begin{array}{l}\text { Asterella multiflora (Steph.) } \\
\text { Pande et al. }\end{array}$ & 1430 & $\mathrm{FC}$ \\
\hline 3. & Aytoniaceae & $\begin{array}{l}\text { Asterella mussuriensis } \\
\text { (Kashyap) Verd. }\end{array}$ & 1250 & $\mathrm{R}$ \\
\hline 4. & Aytoniaceae & $\begin{array}{l}\text { Asterella wallichiana (Lehm. \& } \\
\text { Linden.) Grolle }\end{array}$ & $1350-1750$ & $\mathrm{FC}$ \\
\hline 5. & Aytoniaceae & $\begin{array}{l}\text { Plagiochasma appendiculatum } \\
\text { Lehm. \& Lindenb. }\end{array}$ & $1300-1700$ & $\mathrm{FC}$ \\
\hline 6. & Aytoniaceae & $\begin{array}{l}\text { Plagiochasma pterospermum C. } \\
\text { Massal. }\end{array}$ & $1350-1750$ & $\mathrm{C}$ \\
\hline 7. & Aytoniaceae & $\begin{array}{l}\text { Reboulia hemispherica (L.) } \\
\text { Raddi }\end{array}$ & $1600-3000$ & $\mathrm{R}$ \\
\hline 8. & Cephaloziellaceae & $\begin{array}{l}\text { Cephaloziella massalongi } \\
\text { (Spruce.) K. Muell. }\end{array}$ & $1550-2000$ & $\mathrm{R}$ \\
\hline 9. & Conocephalaceae & $\begin{array}{l}\text { Conocephalum conicum (L.) } \\
\text { Underw. }\end{array}$ & $1890-3800$ & $\mathrm{FC}$ \\
\hline
\end{tabular}


Appendix 1 Contd ...

\begin{tabular}{|c|c|c|c|c|}
\hline 10. & Conocephalaceae & $\begin{array}{l}\text { Conocephalum japonicum } \\
\text { (Thunb.) Grolle }\end{array}$ & 1800 & $\mathrm{R}$ \\
\hline 11. & Frullaniaceae & Frullania ericoides (Nees) Montin & 1740 & $\mathrm{R}$ \\
\hline 12. & Frullaniaceae & Frullania muscicola Steph. & $1280-1840$ & $\mathrm{FC}$ \\
\hline 13. & Frullaniaceae & Frullania retusa Mitt. & 1720 & $\mathrm{FC}$ \\
\hline 14. & Frullaniaceae & Frullania tamarisci (L.) Dumort. & $1480-1840$ & $\mathrm{FC}$ \\
\hline 15. & Frullaniaceae & Frullania yunnanensis Steph. & 2400 & $\mathrm{FC}$ \\
\hline 16. & Geocalycaceae & $\begin{array}{l}\text { Heteroscyphus argutus (Reinw. } \\
\text { et al.) Schiffn, }\end{array}$ & $1300-1800$ & $\mathrm{R}$ \\
\hline 17. & Geocalycaceae & $\begin{array}{l}\text { Heteroscyphus coliatus (Hook.) } \\
\text { Schiffn. }\end{array}$ & 3000 & $\mathrm{R}$ \\
\hline 18. & Geocalycaceae & $\begin{array}{l}\text { Heteroscyphus planus (Mitt.) } \\
\text { Schiff. }\end{array}$ & 1300 & $\mathrm{R}$ \\
\hline 19. & Jungermanniaceae & $\begin{array}{l}\text { Jamesoniella automnalis (D.C) } \\
\text { Steph. }\end{array}$ & 2440 & VR \\
\hline 20. & Jungermanniaceae & Jungermannia appressifolia Mitt. & 2800 & $\mathrm{FC}$ \\
\hline 21. & Jungermanniaceae & $\begin{array}{l}\text { Jungermannia atrovirens } \\
\text { Dumort. }\end{array}$ & $1250-2800$ & $\mathrm{FC}$ \\
\hline 22. & Jungermanniaceae & Jungermannia hyalina Lyell & 3500 & $\mathrm{R}$ \\
\hline 23. & Jungermanniaceae & $\begin{array}{l}\text { Jungermannia tetragona } \\
\text { Lindenb. }\end{array}$ & $1200-1300$ & $\mathrm{FC}$ \\
\hline 24. & Lejeuneaceae & Lejeunea cavifolia (Ehrh.) Lindb. & $1766-1950$ & $\mathrm{FC}$ \\
\hline 25. & Lejeuneaceae & Lejeunea flava (Sw.) Nees & 2420 & $\mathrm{FC}$ \\
\hline 26. & Lepidoziaceae & $\begin{array}{l}\text { Bazzania tridens (Reinw. et al.) } \\
\text { Trev. }\end{array}$ & 2440 & $\mathrm{R}$ \\
\hline 27. & Lepidoziaceae & Lepidozia reptans (L.) Dumort. & $2800-3000$ & $\mathrm{FC}$ \\
\hline
\end{tabular}


J. Nat. Hist. Mus. Vol. 27, 2013

Appendix 1 Contd ...

\begin{tabular}{|c|c|c|c|c|}
\hline 28. & Marchantiaceae & $\begin{array}{l}\text { Marchantia emarginata Reinw. } \\
\text { et al. }\end{array}$ & $1200-1800$ & $\mathrm{C}$ \\
\hline 29. & Marchantiaceae & Marchantia paleacea Bertol. & $1300-1800$ & $\mathrm{R}$ \\
\hline 30. & Marchantiaceae & Marchantia polymorpha L. & $1300-1900$ & $\mathrm{C}$ \\
\hline 31. & Pelliaceae & Pellia epiphylla (L.) Corda & $2420-2800$ & $\mathrm{R}$ \\
\hline 32. & Plagiochileaceae & Plagiochila chinensis Steph. & 2460 & $\mathrm{R}$ \\
\hline 33. & Plagiochileaceae & Plagiochila cuspidata Steph. & $2800-3800$ & $\mathrm{FC}$ \\
\hline 34. & Plagiochileaceae & Plagiochila denticulata Mitt. & $2400-3850$ & $\mathrm{FC}$ \\
\hline 35. & Plagiochileaceae & Plagiochila flexuosa Mitt. & 2350 & $\mathrm{R}$ \\
\hline 36. & Plagiochileaceae & Plagiochila retusa Mitt. & 2400 & $\mathrm{R}$ \\
\hline 37. & Porellaceae & Porella arboria (Taylor) Spruce & 2300 & $\mathrm{FC}$ \\
\hline 38. & Porellaceae & $\begin{array}{l}\text { Porella denticulata (Kashyap \& } \\
\text { R.S. Chopra) J.X. Luo }\end{array}$ & 2120 & $\mathrm{FC}$ \\
\hline 39. & Porellaceae & Porella nitens (Steph.) S. Hatt. & 1700 & $\mathrm{R}$ \\
\hline 40. & Ricciaceae & Riccia himalayensis Steph. & 1720 & $\mathrm{R}$ \\
\hline 41. & Scapaniaceae & Scapania ciliata Sande Lac. & 2400 & $\mathrm{R}$ \\
\hline 42. & Scapaniaceae & Scapania stephani K. Muell. & 2460 & $\mathrm{R}$ \\
\hline 43. & Targioniaceae & $\begin{array}{l}\text { Cyathodium cavernarum (Kunze) } \\
\text { Lehm. }\end{array}$ & 900 & $\mathrm{R}$ \\
\hline 44. & Targioniaceae & Cyathodium tuberosum Kashyap & $850-1766$ & $\mathrm{R}$ \\
\hline 45 & Targioniaceae & Targionia hypophylla L. & $1300-1840$ & $\mathrm{C}$ \\
\hline 46. & Trichocoleaceae & $\begin{array}{l}\text { Trichocolea tomentaella (Furh.) } \\
\text { Dumort. }\end{array}$ & $2440-2460$ & $\mathrm{FC}$ \\
\hline 47. & Wiesnerellaceae & Dumortiera hirsuta (SW.) Nees & $1350-2440$ & $\mathrm{FC}$ \\
\hline 48. & Wiesnerellaceae & Wiesnerella denudata Steph. & 1700 & $\mathrm{R}$ \\
\hline
\end{tabular}




\section{Musci}

\begin{tabular}{|c|c|c|c|c|}
\hline 1. & Amblystegaceae & $\begin{array}{l}\text { Calliergonella cuspida (Hedw.) } \\
\text { Loesk. }\end{array}$ & 1800 & $\mathrm{FC}$ \\
\hline 2. & Amblystegaceae & Campylium halleri Hedw. & 1800 & $\mathrm{R}$ \\
\hline 3. & Bartramiaceae & $\begin{array}{l}\text { Fleischerobryum longicollis } \\
\text { (Hampe) Loesk. }\end{array}$ & 1830 & $\mathrm{R}$ \\
\hline 4. & Bartramiaceae & Philinotis fontana (Hedw.) Brid. & $1650-2420$ & $\mathrm{FC}$ \\
\hline 5. & Bartramiaceae & $\begin{array}{l}\text { Philinotis hastata (Duby) Wijk \& } \\
\text { Margad }\end{array}$ & 1600 & $\mathrm{R}$ \\
\hline 6. & Bartramiaceae & Philinotis thwaitsii Mitt. & $1760-1850$ & $\mathrm{FC}$ \\
\hline 7. & Brachytheciaceae & $\begin{array}{l}\text { Brachythecium buchananii } \\
\text { (Hook.) A. Jaeger }\end{array}$ & $1900-2000$ & $\mathrm{FC}$ \\
\hline 8. & Brachytheciaceae & Bryhnia decurvans (Mitt.) Dixon & $2240-2800$ & $\mathrm{R}$ \\
\hline 9. & Brachytheciaceae & $\begin{array}{l}\text { Brachythecium recurvirostrum } \\
\text { (Hedw.) Chen }\end{array}$ & 3800 & $\mathrm{FC}$ \\
\hline 10. & Brachytheciaceae & $\begin{array}{l}\text { Eurhynchium proelongum } \\
\text { (Hedw.) Schimp. var. rigidum }\end{array}$ & $1295-1816$ & $\mathrm{FC}$ \\
\hline 11. & Brachytheciaceae & $\begin{array}{l}\text { Eurhynchium ripariodes (Hedw.) } \\
\text { Richs. }\end{array}$ & $1750-1825$ & $\mathrm{C}$ \\
\hline 12. & Brachytheciaceae & $\begin{array}{l}\text { Homalothecium nigheriensis } \\
\text { (Mont.) H. Rob. }\end{array}$ & 2400 & $\mathrm{R}$ \\
\hline 13. & Bryaceae & $\begin{array}{l}\text { Anomobryum auratum (Mitt.) A. } \\
\text { Jaeger }\end{array}$ & $2300-2420$ & $\mathrm{C}$ \\
\hline 14. & Bryaceae & Brachymnium acuminatum Harv. & 1550 & $\mathrm{FC}$ \\
\hline 15. & Bryaceae & $\begin{array}{l}\text { Brachymnium exile (Dozy \& } \\
\text { Molk.) Bosch \& Lacey }\end{array}$ & 2350 & $\mathrm{R}$ \\
\hline 16. & Bryaceae & Brachymnium nepalensis Hook. & 1560 & $\mathrm{R}$ \\
\hline
\end{tabular}


Appendix 1 Contd ...

\begin{tabular}{|c|c|c|c|c|}
\hline 17. & Bryaceae & $\begin{array}{l}\text { Brachymnium ptychothecium } \\
\text { (Besch.) Ochi }\end{array}$ & 1825 & $\mathrm{R}$ \\
\hline 18. & Bryaceae & Bryum argenteum Hedw. & $1300-3900$ & $\mathrm{C}$ \\
\hline 19. & Bryaceae & Bryum cellulare Hook. & 3100 & $\mathrm{R}$ \\
\hline 20. & Bryaceae & $\begin{array}{l}\text { Bryum clavatum (Schimp.) C. } \\
\text { Muell. }\end{array}$ & 3000 & $\mathrm{R}$ \\
\hline 21. & Bryaceae & Bryum coronatum Schwaegr. & $1250-1760$ & $\mathrm{R}$ \\
\hline 22. & Bryaceae & $\begin{array}{l}\text { Bryum pseudotriquestrum } \\
\text { (Hedw.) Gaertn. }\end{array}$ & 4250 & $\mathrm{R}$ \\
\hline 23. & Bryaceae & Bryum recurvulum Mitt. & 4300 & $\mathrm{R}$ \\
\hline 24. & Bryaceae & $\begin{array}{l}\text { Pohlia acuminata Hoppe \& } \\
\text { Hornsch. }\end{array}$ & 4150 & $\mathrm{R}$ \\
\hline 25. & Bryaceae & Pohlia elongata Hedw. & $1750-3900$ & $\mathrm{MC}$ \\
\hline 26. & Bryaceae & $\begin{array}{l}\text { Pohlia leucoloma (Bosch. \& } \\
\text { Lacey) Fleisch. }\end{array}$ & $1300-2500$ & $\mathrm{FC}$ \\
\hline 27. & Bryaceae & Pohlia nutans (Hedw.) Lindb & $2360-2440$ & $\mathrm{R}$ \\
\hline 28. & Bryaceae & $\begin{array}{l}\text { Rhodobryum giganteum } \\
\text { (Schwaegr.) Paris }\end{array}$ & $2460-3800$ & $\mathrm{C}$ \\
\hline 29. & Bryaceae & $\begin{array}{l}\text { Rhodobryum roseum (Hedw.) } \\
\text { Limpr. }\end{array}$ & $1765-3800$ & $\mathrm{FC}$ \\
\hline 30. & Calymperaceae & Calymperes erosum $\mathrm{C}$. Muell. & 1720 & $\mathrm{C}$ \\
\hline 31. & Calymperaceae & $\begin{array}{l}\text { Syrrhopodon gardneri (Hook.) } \\
\text { Schwaegr. }\end{array}$ & 1650 & $\mathrm{C}$ \\
\hline 32. & Campylopodiaceae & $\begin{array}{l}\text { Campylopodium khasianum } \\
\text { (Griff.) Paris }\end{array}$ & $2100-4000$ & $\mathrm{R}$ \\
\hline 34. & Dicranaceae & $\begin{array}{l}\text { Campylopus ericoides (Griff.) A. } \\
\text { Jaeger }\end{array}$ & 1360 & $\mathrm{FC}$ \\
\hline 35. & Dicranaceae & Campylopus rechardii Brid. & $1934-2120$ & VR \\
\hline
\end{tabular}


Appendix 1 Contd ...

\begin{tabular}{|c|c|c|c|c|}
\hline 36. & Dicranaceae & $\begin{array}{l}\text { Campylopus subfragilis Ren. \& } \\
\text { Cardot }\end{array}$ & 1770 & $\mathrm{R}$ \\
\hline 37. & Dicranaceae & $\begin{array}{l}\text { Dicranella heteromalla (Hedw.) } \\
\text { Schimp. }\end{array}$ & 1940 & $\mathrm{R}$ \\
\hline 38. & Dicranaceae & Dicranum himalayanum Mitt. & 2850 & $\mathrm{R}$ \\
\hline 39. & Dicranaceae & $\begin{array}{l}\text { Symblepahris reinwardtii (Dozy } \\
\text { \& Molk.) Mitt. }\end{array}$ & $3500-4000$ & $\mathrm{FC}$ \\
\hline 40. & Dicranaceae & Trematodon longicolle Michx. & $1600-2160$ & $\mathrm{C}$ \\
\hline 41. & Entodontaceae & $\begin{array}{l}\text { Entodon flavescens (Hedw.) A. } \\
\text { Jaeger }\end{array}$ & 2050 & $\mathrm{FC}$ \\
\hline 42. & Entodontaceae & $\begin{array}{l}\text { Entodon rubicundus (Mitt.) A. } \\
\text { Jaeger }\end{array}$ & $1400-2220$ & $\mathrm{R}$ \\
\hline 43. & Entodontaceae & Entodon veridulus Cardot & 2430 & $\mathrm{FC}$ \\
\hline 44. & Entodontaceae & $\begin{array}{l}\text { Erythrodontium julaceum (Hook. } \\
\text { ex Schwaegr.) Paris }\end{array}$ & 1760 & $\mathrm{FC}$ \\
\hline 45. & Fissidentaceae & Fissidens cristatus Wils. ex Mitt. & 2440 & $\mathrm{FC}$ \\
\hline 46. & Fissidentaceae & $\begin{array}{l}\text { Fissidens ceylonensis Dozy \& } \\
\text { Molk. }\end{array}$ & $1900-2200$ & $\mathrm{FC}$ \\
\hline 47. & Fissidentaceae & Fissidens grandifrons Brid. & $2500-2550$ & $\mathrm{FC}$ \\
\hline 48. & Fissidentaceae & Fissidens nobilis Griff. & 1300 & $\mathrm{FC}$ \\
\hline 49. & Fissidentaceae & Fissidens sylvaticus Griff. & 1750 & $\mathrm{R}$ \\
\hline 50. & Fissidentaceae & Fissidens taxifolius Hedw. & $2000-2900$ & $\mathrm{R}$ \\
\hline 51. & Funariaceae & Funaria hygrometrica Hedw. & $1300-3800$ & $\mathrm{C}$ \\
\hline 52. & Grimmiaceae & Grimmia affinis Hornsch. & 3450 & $\mathrm{FC}$ \\
\hline 53. & Grimmiaceae & Grimmia ovalis (Hedw.) Lindb. & 3400 & $\mathrm{R}$ \\
\hline 54. & Hylocomiaceae & $\begin{array}{l}\text { Leptohymenium tenue (Hook.) } \\
\text { Schwaegr. }\end{array}$ & 2460 & $\mathrm{R}$ \\
\hline
\end{tabular}


J. Nat. Hist. Mus. Vol. 27, 2013

Appendix 1 Contd ...

\begin{tabular}{|c|c|c|c|c|}
\hline 55. & Hypnaceae & $\begin{array}{l}\text { Ectropothecium sikkimense } \\
\text { (Ren. \& Cardot) Ren. \& Cardot }\end{array}$ & 2000 & $\mathrm{FC}$ \\
\hline 56. & Hypnaceae & Ectropothecium nervosum Dixon & 1765 & $\mathrm{R}$ \\
\hline 56. & Hypnaceae & $\begin{array}{l}\text { Hypnum pleumaforme W. } \\
\text { Wilson }\end{array}$ & $1800-4000$ & $\mathrm{C}$ \\
\hline 57. & Hypnaceae & $\begin{array}{l}\text { Isopterigium albiscens (Hook.) } \\
\text { A. Jarger }\end{array}$ & $1200-1300$ & $\mathrm{FC}$ \\
\hline 58. & Hypnaceae & $\begin{array}{l}\text { Ptilium crista-castrensis (Hedw.) } \\
\text { De Not. }\end{array}$ & $1700-4250$ & $\mathrm{R}$ \\
\hline 59. & Hypnaceae & $\begin{array}{l}\text { Taxiphyllum taxirameum (Mitt.) } \\
\text { Fleisch. }\end{array}$ & $1410-2700$ & $\mathrm{FC}$ \\
\hline 60. & Leucobryaceae & $\begin{array}{l}\text { Leucobryum juniperoideum } \\
\text { (Brid.) C. Muell. }\end{array}$ & 2500 & $\mathrm{FC}$ \\
\hline 61. & Leucobryaceae & Leucobryum mittenii Besch. & 900 & $\mathrm{FC}$ \\
\hline 62. & Leucobryaceae & Octoblepharum albidum Hedw. & $1200-1300$ & $\mathrm{FC}$ \\
\hline 63. & Meteoriaceae & $\begin{array}{l}\text { Barbella stevensii (Ren. \& } \\
\text { Cardot) M. Fleisch. }\end{array}$ & $1720-2500$ & $\mathrm{FC}$ \\
\hline 64. & Mniaceae & Mnium laevinerve Cardot & 2600 & $\mathrm{R}$ \\
\hline 65. & Mniaceae & Mnium lycopodioides Schwaegr. & 3000 & $\mathrm{C}$ \\
\hline 66. & Mniaceae & Mnium punctatum Hedw. & $2800-3850$ & $\mathrm{FC}$ \\
\hline 67. & Mniaceae & Mnium rostratum Schrad. & 1890 & $\mathrm{R}$ \\
\hline 68. & Mniaceae & $\begin{array}{l}\text { Orthomnium bryoides (Griff.) } \\
\text { Norkett }\end{array}$ & 1820 & $\mathrm{R}$ \\
\hline 69. & Plagiomniaceae & $\begin{array}{l}\text { Plagiomnium cuspidatum } \\
\text { (Hedw.) T. Kop. }\end{array}$ & 2650 & $\mathrm{R}$ \\
\hline 70. & Plagiomniaceae & $\begin{array}{l}\text { Plagiomnium succulenteum } \\
\text { (Wils.) Lindb. }\end{array}$ & 1250 & $\mathrm{FC}$ \\
\hline 71. & Plagiotheciaceae & $\begin{array}{l}\text { Plagiothecium nemorale (Mitt.) } \\
\text { A. Jaeger }\end{array}$ & 1766 & $\mathrm{FC}$ \\
\hline
\end{tabular}


Appendix 1 Contd ...

\begin{tabular}{|c|c|c|c|c|}
\hline 72. & Polytrichaceae & $\begin{array}{l}\text { Pogonatum aloides (Hedw.) P. } \\
\text { Beauv. }\end{array}$ & 1700 & $\mathrm{R}$ \\
\hline 73. & Polytrichaceae & $\begin{array}{l}\text { Pogonatum junghunianum (Dozy } \\
\text { \& Molk.) Dozy \& Molk. }\end{array}$ & 1300 & $\mathrm{FC}$ \\
\hline 74. & Polytrichaceae & $\begin{array}{l}\text { Pogonatum microstomum } \\
\text { (Schwaegr.) Brid. }\end{array}$ & $1480-2060$ & $\mathrm{MC}$ \\
\hline 75. & Polytrichaceae & $\begin{array}{l}\text { Pogonatum microphyllum (Dozy } \\
\text { \& Molk.) Dozy \& Molk, }\end{array}$ & $1950-3800$ & $\mathrm{C}$ \\
\hline 76. & Polytrichaceae & Pogonatum nudiusculum Mitt. & 2040 & $\mathrm{FC}$ \\
\hline 77. & Polytrichaceae & $\begin{array}{l}\text { Pogonatum submacrophyllum } \\
\text { Herzog. }\end{array}$ & $2460-2550$ & $\mathrm{FC}$ \\
\hline 78. & Polytrichaceae & Polytrichum commune Hedw. & $1760-2800$ & $\mathrm{FC}$ \\
\hline 79. & Polytrichaceae & Polytrichum urnigerum Hedw. & 2600 & $\mathrm{FC}$ \\
\hline 80. & Pottiaceae & Barbula constricta Mitt. & 2800 & $\mathrm{R}$ \\
\hline 81. & Pottiaceae & Barbula tenuirostries Brid. & $1760-1950$ & $\mathrm{FC}$ \\
\hline 82. & Pottiaceae & $\begin{array}{l}\text { Bryoerythrophyllum } \\
\text { nogochianum Gangulee }\end{array}$ & 2460 & $\mathrm{FC}$ \\
\hline 83. & Pottiaceae & $\begin{array}{l}\text { Bryoerythrophyllum recurvirostre } \\
\text { (Hedw.) P.C. Chen }\end{array}$ & 2420 & $\mathrm{FC}$ \\
\hline 84. & Pottiaceae & $\begin{array}{l}\text { Hydrogonium arcuatum (Griff.) } \\
\text { Wijk. \& Margad }\end{array}$ & 1480 & $\mathrm{FC}$ \\
\hline 85. & Pottiaceae & $\begin{array}{l}\text { Hyophila involuta (Hook.) A. } \\
\text { Jaeger }\end{array}$ & $1510-2500$ & $\mathrm{FC}$ \\
\hline 86. & Pottiaceae & $\begin{array}{l}\text { Oxystegus cylindricus (Brid.) } \\
\text { Hilp. ex Beih. }\end{array}$ & $1755-2449$ & $\mathrm{C}$ \\
\hline 87. & Sphagnaceae & $\begin{array}{l}\text { Sphagnum cuspidatulum C. } \\
\text { Muell. }\end{array}$ & $2100-3000$ & $\mathrm{FC}$ \\
\hline 88. & Sphagnaceae & $\begin{array}{l}\text { Sphagnum junghuhnianum Dozy } \\
\text { \& Molk. }\end{array}$ & 2200 & $\mathrm{R}$ \\
\hline
\end{tabular}




\section{Appendix 1 Contd ...}

\begin{tabular}{|l|l|l|l|l|}
\hline 89. & Splachnaceae & Tayloria indica Mitt. & 2460 & $\mathrm{R}$ \\
\hline 90. & Stereophyllaceae & $\begin{array}{l}\text { Entodontopsis leucostega (Brid.) } \\
\text { W.R. Buck \& Ireland }\end{array}$ & 1750 & $\mathrm{R}$ \\
\hline 91. & Stereophyllaceae & $\begin{array}{l}\text { Entodontopsis tavoyense (Hook. } \\
\text { F.) W. R. Buck \& Ireland }\end{array}$ & 1816 & $\mathrm{MC}$ \\
\hline 92. & Stereophyllaceae & $\begin{array}{l}\text { Entodontopsis wightii (Mit.) W. } \\
\text { R. Buck \& Ireland }\end{array}$ & 2000 & $\mathrm{C}$ \\
\hline 93. & Thuidaceae & $\begin{array}{l}\text { Actinothuidium hookeri (Mitt.) } \\
\text { Broth. }\end{array}$ & 3600 & $\mathrm{R}$ \\
\hline 94. & Thuidaceae & $\begin{array}{l}\text { Haplocladium angustifolium } \\
\text { (Hampe. \& C.Muell.) Broth. }\end{array}$ & 1350 & $\mathrm{R}$ \\
\hline 95. & Thuidaceae & $\begin{array}{l}\text { Herpetineurion toccoae (Sull.\& } \\
\text { Lesq.) Cardot }\end{array}$ & $1350-3200$ & $\mathrm{FC}$ \\
\hline 96. & Thuidaceae & $\begin{array}{l}\text { Thuidium cambifolium (Dozy \& } \\
\text { Molk.) Dozy \& Molk. }\end{array}$ & $1880-4100$ & $\mathrm{C}$ \\
\hline 97. & Thuidaceae & $\begin{array}{l}\text { Thuidium glaucinum (Mitt.) } \\
\text { Bosch. }\end{array}$ & $\begin{array}{l}\text { Thuidium tamariscellum (C. } \\
\text { Muell.) Bosch. \& Lacey }\end{array}$ & 1500 \\
\hline 98. & Thuidaceae & $\mathrm{CC}$ \\
\hline
\end{tabular}

$\mathrm{C}=$ common; $\mathrm{FC}=$ fairly common; $\mathrm{MC}=$ most common; $\mathrm{R}=$ rare $; \mathrm{VR}=$ very rare

\section{AUTHOR'S ADDRESS}

\section{Nirmala Pradhan}

(email: bryonep@yahoo.com)

Natural History Museum, Tribhuvan University

Swayambhu, Kathmandu, Nepal 